\title{
Abrasive WaterJet Machining of Thick Carrara Marble: Cutting Performance vs. Profile, Lagging and WaterJet Angle Assessments
}

\author{
Mohammad S. Alsoufi, Dhia K. Suker, Mohammed W. Alhazmi, Sufyan Azam \\ Department of Mechanical Engineering, College of Engineering and Islamic Architecture, Umm Al-Qura University, Makkah, \\ KSA \\ Email:mssoufi@uqu.edu.sa
}

How to cite this paper: Alsoufi, M.S., Suker, D.K., Alhazmi, M.W. and Azam, S. (2017) Abrasive WaterJet Machining of Thick Carrara Marble: Cutting Performance vs. Profile, Lagging and WaterJet Angle Assessments. Materials Sciences and Applications, 8, 361-375.

https://doi.org/10.4236/msa.2017.85025

Received: April 21, 2017

Accepted: May 22, 2017

Published: May 25, 2017

Copyright $\odot 2017$ by authors and Scientific Research Publishing Inc. This work is licensed under the Creative Commons Attribution International License (CC BY 4.0).

http://creativecommons.org/licenses/by/4.0/

(c) $\underset{\mathrm{EY}}{0}$ Open Access

\begin{abstract}
This paper deals with an assessment of the machined surface created by abrasive waterjet technology regarding its cutting performance versus profile, lagging and waterjet angle assessments. The results of the experiments presented in this study are with regard to Carrara marble. The machined surfaces were measured in seven different locations across a $40 \mathrm{~mm}$ depth of cut by a high precision contact-type profilometer and thus assessed using the standardized amplitude parameters of the profile distribution. The lagging and waterjet angle were also evaluated by creating a digital photo of the machined surface together with a reference gauge. The existence of machining marks on the machined surface has been mostly noticeable in the bottom zone around $\sim 20 \mathrm{~mm}$ depth of cut down to jet exit. This investigation leads to a conclusion that, stand-off distance and traverse rate play the roles of the utmost importance in considerations of the machined surface quality in contrast to abrasive mass flow rate. In addition, while the striation zone (rough surface) cannot be eliminated entirely, by selecting proper process parameters, a smooth cutting machined surface can be accomplished.
\end{abstract}

\section{Keywords}

Skewness, Kurtosis, Lagging, Waterjet Angle, Abrasive WaterJet, Carrara Marble

\section{Introduction}

The machined surface quality control of Carrara marble is a very significant part of the surface preparation in all categories of technologies that are used in Al-Masjid Al-Haram, The Holy Mosque, in Makkah, KSA, for internal flooring 
and also for internal and external cladding applications [1]. Since 1930, when topography meters's test-rig was invented, the machined surface quality control and its measurement have become the most critical factors and significant functionality indicators in the repeatability and reproducibility performance of highprecision applications while also minimizing the negative impact on the environment [2]. New manufacturing procedures and measurement methods, which provide us with new and improved machined surface properties and extensive information about the newly created surfaces are implemented into everyday practice. The current trends in environmental protection contribute to the development of non-conventional machining (NCM) methods, which include abrasive waterjet technology [3]. Abrasive waterjet (AWJ) technology is a material removal process [4] [5]. It is a state-of-the-art non-traditional machining (NTM) process [6] [7]. This technology generates a special surface on the machined material, which should be studied precisely when assessing the parameters of the surface topography. Figure 1 shows the schematic illustration of an abrasive waterjet machine along with the material to be cut. In this schematic diagram of an AWJ machine, the water with a high-pressure of up to $400 \mathrm{MPa}$ is focused through a sapphire orifice, thus producing a high-velocity jet that passes through a mixing chamber and hits the target material to be cut. The abrasive particles' agents are then supplied through the vacuum formed in the mixing chamber. Momentum transfer from the jet to the abrasive particles takes place within a narrow mixing tube, also called the nozzle. Finally, the high-pressure water jet charged with the abrasive particles agent then cut through the target material [8]. Water mixed with debris is eventually collected, filtered and dried in order to re-use the abrasive particle agent again. The recycling of the abrasive particles' agent makes the AWJ technology a clear example of greener manufacturing, cleaner production, and a generally more economical and more sustainable method [9]. The main advantage of AWJ technology is the elimination of the thermal effects due to the cutting process of using the medium formed of fluid on the workpiece so that there is no thermal degradation and it is not

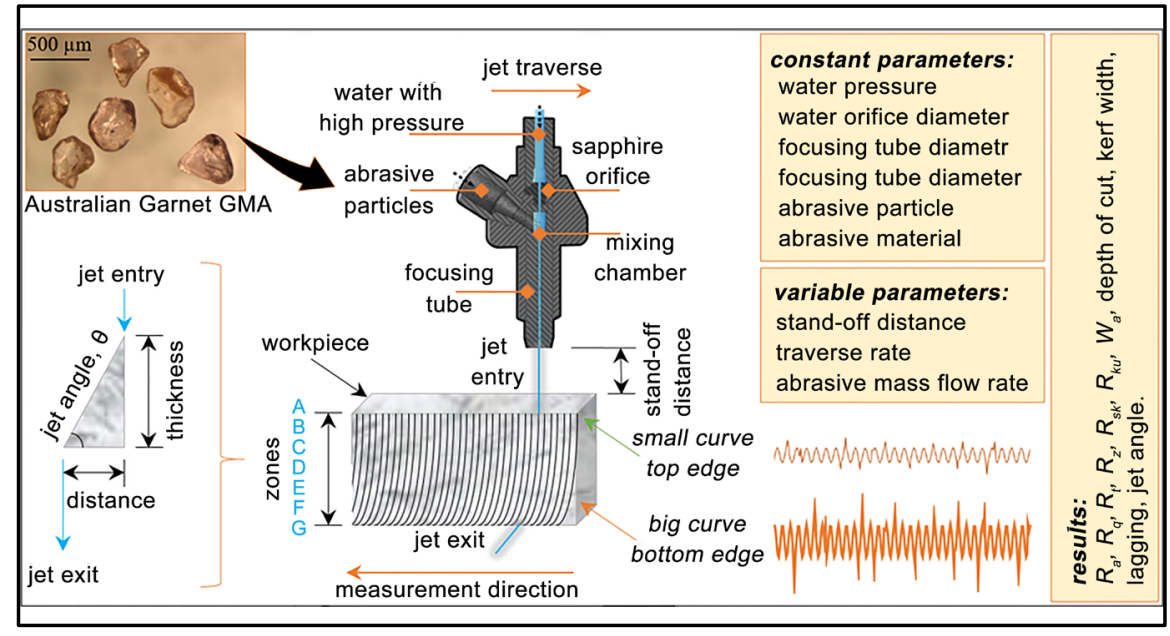

Figure 1. Schematic of abrasive waterjet machine along with target material to be cut. 
flammable [10]. The AWJ machine can disintegrate almost all engineering industrial materials, ranging from a soft material such as wood, plastics, rubber, and so on, to hard materials such as Inconel, titanium, and so on [11].

Published analyses and repeatability performance data relating to the effects of the stand-off distance (SOD), traverse rate (TR) and abrasive mass flow rate (AMFR) machining process factors on the surface roughness, waviness and profile, e.g. [6]. However, we found no authoritative public data on skewness, $R_{\mathrm{sk}}$, and kurtosis, $R_{\mathrm{ku}}$, performance. Therefore, we report on an experimental study to examine the effects of AWJ variables on skewness, $R_{\mathrm{sk}}$, and kurtosis, $R_{\mathrm{ku}}$, in AWJ cutting of Carrara marble material. Lagging and waterjet angle assessments are also examined.

\subsection{Surface Profile Assessment}

The most frequently-used roughness parameter applied for the evaluation of machined surface materials is the amplitude parameter, $R_{\mathrm{a}}$ and indeed, it is the universally recognized one. It is the arithmetical mean of the absolute value of $z(x)$ departures of the roughness profile from the mean line, 1 ; see Equation (1) [12] [13]. However, the amplitude parameter, $R_{\mathrm{a}}$, does not react sensitively to height variations of the assessed machined surface profile, as it is crucial to study the height variation after the abrasive waterjet machine cuts the target material. For this reason, the parameters $R_{\mathrm{q}}$ (see Equation (2), the root mean square RMS to $R_{\mathrm{a}}$ ) was observed as the amplitude parameters for more detailed machined surface evaluation since they respond more sensitively to local inequalities of the assessed machined surface [5]. They provide more precise information about the studied surface texture of Carrara marble used in this investigation.

$$
\begin{gathered}
R_{a}=\frac{1}{l} \int_{0}^{l}|z(x)| \mathrm{d} x \\
R_{q}=\sqrt{\frac{1}{l} \int_{0}^{l}\left|z^{2}(x)\right| \mathrm{d} x}
\end{gathered}
$$

Skewness, $R_{\mathrm{sk}}$, as stated in Equation (3), is defined by ISO 4287 (1997) [14], as a measure of the degree of asymmetry of the amplitude density curve and is characterized as positive or negative distributions. Empty machined surfaces of material present positive skewness distribution, while full machined surfaces exhibit negative skewness distribution, see Figure 2 [14]. It is a significant amplitude parameter for tribological applications, such as bearing surface functionality, friction, wear control and so on. Mathematically, it is the third moment of height amplitude distribution and is normalized by the standard deviation. It acquires a null value for an entirely random machined surface profile due to uniform distribution of profile amplitudes about the mean line. However, it is an important measure in categorizing the manufacturing processes as it reflects the distribution and shape of the unit event(s). A positively skewed profile distribution (large tail to the right) exhibits excellent gripping and locking properties in metallic materials and helps in improving the fatigue life of components, where cracks may initiate from surface defects. A negatively skewed profile distribution 
(large tail to the left) indicates a surface with excellent bearing properties due to valleys acting as lubrication reservoirs.

Kurtosis, $R_{\mathrm{ku}}$, as stated in Equation (4), is defined by ISO 4287 (1997) [14], as a method of quantifying bearing properties by measuring the degree of peakedness of the amplitude density curve and takes the value 3 for normal distribution, see Figure 3 [14]. Mathematically, it is the fourth moment of height amplitude distribution and is also normalized by the standard deviation. It indicates narrowness and/or widths of the probability density function in comparison to the normal distribution (Gaussian) of the profile coordinates, where parameter $R_{\mathrm{ku}}=3$ (mesokurtic). A profile with sharper peaks (spiky) has the value of $R_{\mathrm{ku}}>$ 3 (leptokurtic, homogeneous and narrow scatter). In contrast, $R_{\mathrm{ku}}<3$ (platykurtic, heterogeneous and wide scatter) indicates a profile distribution with bumpy, round and less frequent peaks. In this regard, information is provided on the real contact area and wears resistance. In addition, it is likely to detect the periodicity of the profile distribution $R_{\mathrm{ku}}<3$.

$$
\begin{aligned}
& R_{s k}=\frac{1}{R_{q}^{3}}\left[\frac{1}{l} \int_{0}^{l} z^{3}(x) \mathrm{d} x\right] \\
& R_{k u}=\frac{1}{R_{q}^{4}}\left[\frac{1}{l} \int_{0}^{l} z^{4}(x) \mathrm{d} x\right]
\end{aligned}
$$

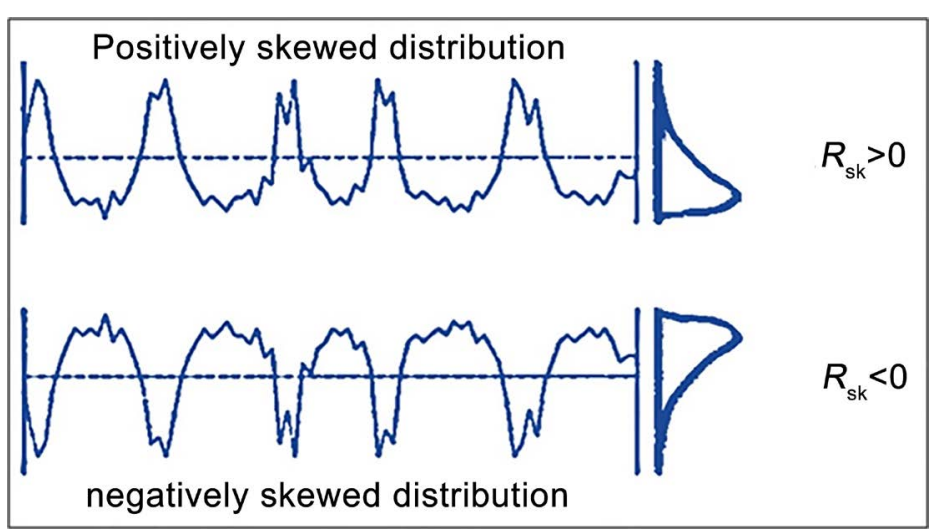

Figure 2. Skewness distribution, adapted from [14].

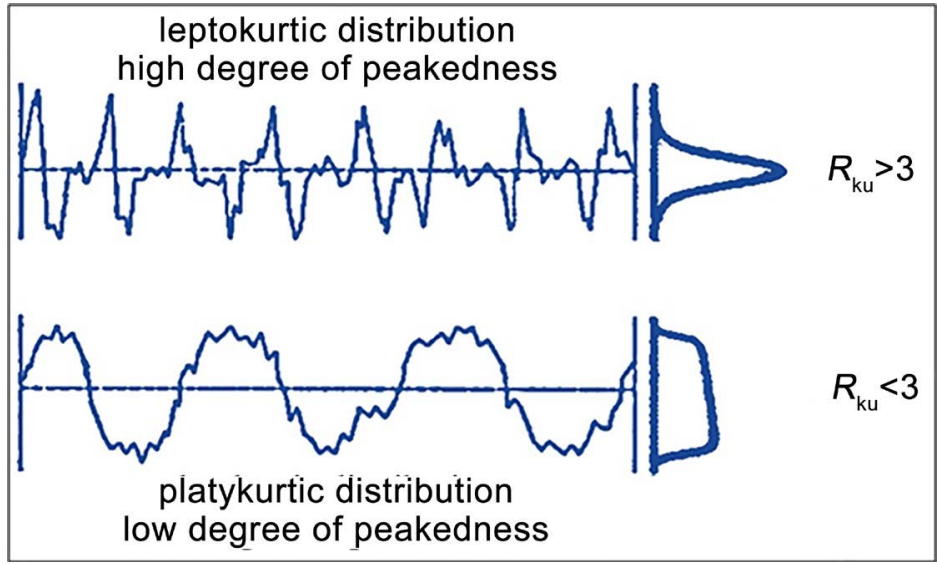

Figure 3. Kurtosis distribution, adapted from [14]. 


\section{Testing Assembly}

Owing to the importance of the process parameters in the AWJ process, standoff distance (SOD), traverse rate (TR) and abrasive mass flow rate (AMFR) have been selected as the actual value of the process parameters for the present study. The Carrara marble material of $100 \mathrm{~mm} \times 10 \mathrm{~mm} \times 40 \mathrm{~mm}$ has been made to cut in order to produce rectangular specimens on the $x-y$ cutting table with an operating area of $2000 \times 2000 \mathrm{~mm}$. Test samples were prepared from a big rock that was free from alteration and fracture zones. Since the trial experiments should ideally be conducted in the medium level rating of energy, water pressure has been selected as $400 \mathrm{MPa}$ with low, medium and high abrasive mass flow rate. The TR has been chosen as $2.0,4.0,6.0,8.0$ and $10 \mathrm{~mm} / \mathrm{min}$. The SOD has been selected as 4.0,8.0,12, 16 and $20 \mathrm{~mm}$. The SOD, TR, and AMFR have been used as variable parameters during the AWJ cutting process. Other parameters such as water orifice diameter of $0.3 \mathrm{~mm}$, focusing tube diameter of $0.8 \mathrm{~mm}$, focusing tube length of $76 \mathrm{~mm}$, and garnet abrasive particles (a mesh size of approximately $180 \mu \mathrm{m}(80 \#)$ ) with an impingement angle of $90^{\circ}$ and density of $4100 \mathrm{~kg} / \mathrm{m}^{3}$ have been used as constant parameters during the operation of AWJ. The same abrasive product was utilized in all cutting procedures.

With a combination of low contact force, high displacement sensitivity and the small radius of a $2 \mu \mathrm{m}$ stylus being used, the surface irregularity distribution was measured using a contact-type Taly-Surf ${ }^{\circledast}$ profilometer (Taylor Hobson Precision, Inc.). The tests were carried out under essentially wear-free conditions with nominal applied loads of $0.7 \mathrm{mN}$, which offered resolution down to $0.8 \mathrm{~nm}$, a measuring range of $12.5 \mathrm{~mm}$ in the $x$-axis, and linear speed from 0 to 0.5 $\mathrm{mm} / \mathrm{s}$. The traces were auto-levelled to a linear least-squares straight line and then filtered with a standard $0.8 \mathrm{~mm}$ cut-off. Details of the surface roughness measurement procedure have been reported elsewhere [15]-[20]. Measuring was conducted in seven zone levels per each sample (a total of 12 Carrara marble samples). Zone (A) was located at $5 \mathrm{~mm}$ from the top edge of the Carrara marble and zone $(G)$ was located at 5 from the bottom edge of the sample. The new zones' (B, C, D, E, F) location was $5 \mathrm{~mm}$ from the previous one. A total of 40 $\mathrm{mm}$ thickness of Carrara marble was examined. The obtained data were analyzed with OriginLab ${ }^{\circledast}$ software. Measurement and resultant assessment of machined surfaces were carried out according to international standards [21] [22] [23] [24].

\section{Results and Discussions}

This research work is an extension of a preliminary study conducted on Carrara marble stones. The experimental set-up and results of the surface roughness, $R_{\mathrm{a}}$, and depth of cut (DoC) have already been reported in [25] and are used here as a reference for further investigation. A stylus profilometer reports $R_{\mathrm{a}}$ values of around $R_{\mathrm{a}} \approx 4 \mu \mathrm{m}$ at the top edge and $R_{\mathrm{a}} \approx 93.7 \mu \mathrm{m}$ at the bottom edge of the machined surface [25]. In the current investigation, cutting parameters' per- 
formance against the surface profile, lagging and waterjet angle assessments were made. These parameters such as SOD, TR and AMFR are the key process parameters of the AWJ technology and were selected in response to suggestions from the industry and due to their everyday use for evaluation of machined surface quality in both research and industrial applications.

As regards stress-strain deformation behaviour, rather high deformation stress occurs at the inlet of the jet to the Carrara marble material. Therefore, the separating cut is relatively smooth at the top edge of the target material. But, the deformation stress-strain, i.e., tool cuttability, which is significantly influenced by the TR decreases with any increase in the DoC [26]. For all machined surface of Carrara marble, two regions were dependent on the kinetic energy level of the jet, namely the smooth zone, and the striation zone. The smooth zone represents the smooth cutting zone, whereas, the striation zone represents the initial damage zone and rough cutting zone.

\section{1. $R_{\mathrm{q}} / R_{\mathrm{a}}$ Ratio Assessment}

Analysis of the surface profiles generated under different abrasive waterjet cutting process conditions is conducted qualitatively in terms of the static characteristics in seven different sub-zone levels, including the surface roughness, $R_{\mathrm{a}}$, (which was published in [25]) and the root mean square, $R_{\mathrm{q}}$, in order to generate the ratio of machined surface profile $R_{\mathrm{q}} / R_{\mathrm{a}}$ with the different process parameters of AWJ technology. During the evaluation, the detailed texture of the newlycreated surface must be considered. The texture is separated into elements based on the spacing between inequalities which vary significantly depending on the DoC. The values of the amplitude parameters in the upper part of the separating cut are lower than the values of the amplitude parameters at the bottom. For this reason, the values of amplitude parameters in seven zones were measured in each sample. The ratio of root means square (RMS), $R_{\mathrm{q}}$, to average surface roughness, $R_{\mathrm{a}}$, for SOD, TR, and AMFR was found to be randomly varying around $R_{\mathrm{q}} / R_{\mathrm{a}}=1.23 \pm 0.04, R_{\mathrm{q}} / R_{\mathrm{a}}=1.25 \pm 0.03, R_{\mathrm{q}} / R_{\mathrm{a}}=1.26 \pm 0.04$, respectively, which depicts the inherent characteristic of the machining process. Besides, the maximum and minimum values of $R_{\mathrm{q}} / R_{\mathrm{a}}$ for SOD, TR, and AMFR was found to be around $\left(R_{\mathrm{q}} / R_{\mathrm{a}}\right)_{\max .}=1.34$ and $\left(R_{\mathrm{q}} / R_{\mathrm{a}}\right)_{\min .}=1.16,\left(R_{\mathrm{q}} / R_{\mathrm{a}}\right)_{\max .}=1.31$ and $\left(R_{\mathrm{q}} / R_{\mathrm{a}}\right)_{\min .}=1.18$ and $\left(R_{\mathrm{q}} / R_{\mathrm{a}}\right)_{\text {max. }}=1.34$ and $\left(R_{\mathrm{q}} / R_{\mathrm{a}}\right)_{\min .}=1.21$, respectively. Hence, the amplitude parameters of the machined surface of Carrara marble were increased with the increasing SOD, TR, and AMFR.

\subsection{Skewness vs, Kurtosis Assessment}

Since the abrasive waterjet procedure involves the generation of a new surface profile through the percussive impact of stochastic abrasive particles, the surface topology was studied with the surface asymmetry (skewness, $R_{\text {sk }}$ ) and the coefficient of its inclination (kurtosis, $R_{\mathrm{ku}}$ ) in all samples of Carrara marble. This is vital to predicting the functional behaviour of the machined surface Carrara marble, such as tribological properties of the surface (which involves the study of the 
friction and wear properties of the material). For evaluation of $R_{\mathrm{sk}}$ and $R_{\mathrm{ku}}$, two lines both from the upper part and from the bottom of the cutting wall were removed in order to remove the effect of penetration and cutting tool exit, i.e. AWJ, into and from the cut target.

Figure 4 shows that the plot of $R_{\text {sk }}$ ( $3^{\text {rd }}$ moment) against $R_{\mathrm{ku}}\left(4^{\text {th }}\right.$ moment) of abrasive waterjet generated profile is centred around the random Gaussian profile, which was the negatively and positively skewed distribution and leptokurtic and platykurtic with a low and high degree of peakedness. As can be seen, a value of zero for skewness and three for kurtosis is typical for a random, Gaussian profile and weakly isotropic. The small arc profile kerfs showed the least tendency for positive skewness when machined with an abrasive waterjet. A negatively skewed profile indicates more resistance to abrasive erosion, which in this case is the reduced cutting efficiency of the jet in the direction of jet penetration. The values of $R_{\mathrm{sk}}$ and $R_{\mathrm{ku}}$ are strongly influenced by AWJ on the surface. Most of the surface texture of the Carrara marble is characterized by small values of $R_{\mathrm{sk}}$ and $R_{\mathrm{ku}}$ parameters. These plots show that the majority of SOF, TR, and AMFR display a negative skewness distribution, $R_{\mathrm{sk}}<0$, close to zero in magnitude, which will be beneficial for a large number of applications. Also, this is typical of the erosive nature of water erosion processes.

Figure 4(a) shows $R_{\mathrm{sk}}$ against $R_{\mathrm{ku}}$ with different SOD process parameters. It shows that the general trend of positive and negative skewness is almost equally distributed over all variable process of SOD with a low and high degree of peakedness. The trend of skewness and kurtosis is in the region of $-0.6 \leq R_{\mathrm{sk}} \leq$ 0.44 (57.7\% negatively skewed distribution and $42.3 \%$ positively skewed distribution) and $1.8 \leq R_{\mathrm{ku}} \leq 4.1$ (30.5\% platykurtic distribution and 69.5\% leptokurtic distribution) with a range of 1.04 and 2.3 for $R_{\mathrm{sk}}$ and $R_{\mathrm{ku}}$, respectively. At a stand-off distance of $20 \mathrm{~mm}$, the data was obtained from zone (A) to zone (G), the range of $R_{\mathrm{sk}}$ from -0.315 to 0.1636 (60.8\% negative skewed distribution and $39.2 \%$ positive skewed distribution) and the range of $R_{\mathrm{ku}}$ from 2.3988 to 2.8681 (100\% low degree of peakedness with platykurtic distribution) were obtained, which represents the lowest value of $R_{\mathrm{sk}}$ and $R_{\mathrm{ku}}$ generated among the range of stand-off distance $(4,8,12,16$ and $20 \mathrm{~mm})$. Since the SOD determines the mechanical force applied to the machined surface in the AWJ machining process, it has a role of the most importance in determining the surface roughness, waviness, and form.

Figure 4(b) shows $R_{\mathrm{sk}}$ against $R_{\mathrm{ku}}$ with different TR process parameters. It shows that the general trend of positive and negative skewness is almost equally distributed over all variable processes of TR with low (platykurtic distribution) and high (leptokurtic distribution) degrees of peakedness. The trend of $R_{\mathrm{sk}}$ and $R_{\mathrm{ku}}$ in the region of $-0.75 \leq R_{\mathrm{sk}} \leq 0.65$ (53.5\% negatively skewed distribution and $46.4 \%$ positively skewed distribution) and $2.0 \leq R_{\mathrm{ku}} \leq 3.8$ (34.5\% platykurtic distribution and $65.5 \%$ leptokurtic distribution) with a range of 1.4 and 1.8 for $R_{\mathrm{sk}}$ and $R_{\mathrm{ku}}$, respectively. At the traverse rate, the data was only obtained in the zone $(\mathrm{A}, \mathrm{B}, \mathrm{C})$ as the other zone area of the Carrara marble surface becomes too rough 

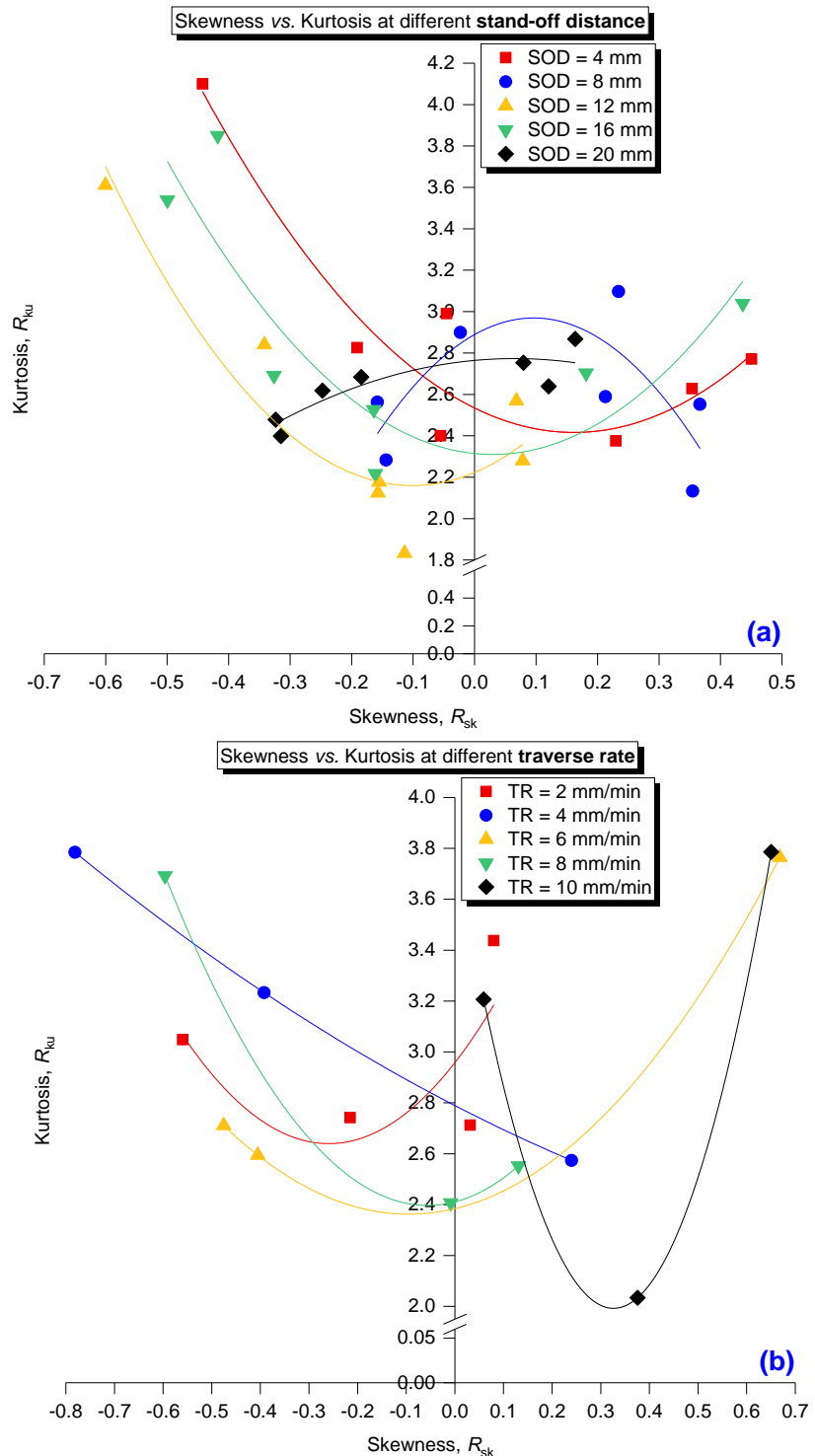

Skewness vs. Kurtosis at different abrasive mass flow rate

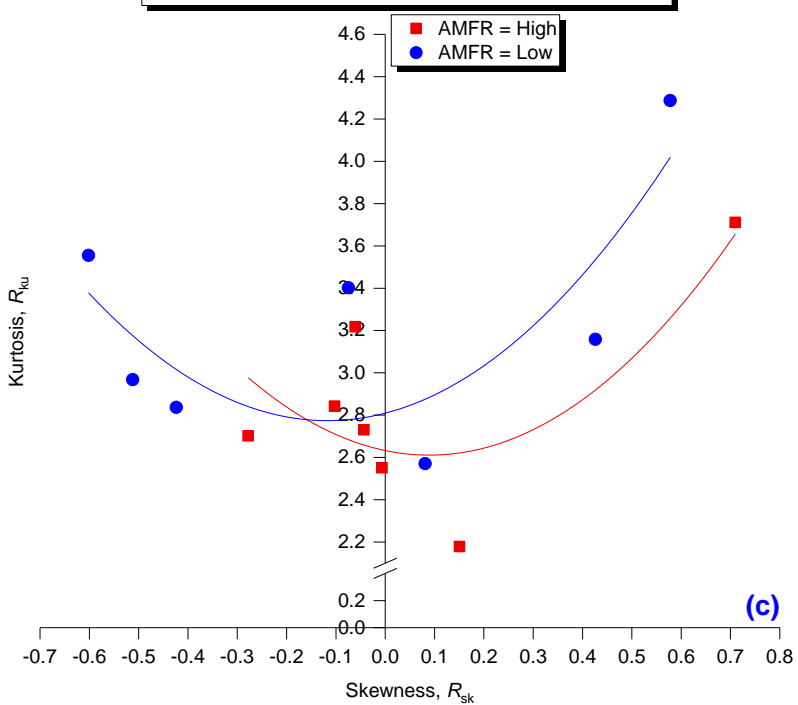

Figure 4. The maps of kurtosis vs. skewness at different process parameters of AWJ. 
and difficult to measure. This is because a faster TR increases the jet deflection which results in a higher magnitude of surface texture profile. At TR $=10 \mathrm{~mm} /$ min, the range of $R_{\mathrm{sk}}$ from 0.3755 to 0.6506 (100\% positively skewed distribution) and the range of $R_{\mathrm{ku}}$ from 2.0338 to 3.7855 (35\% platykurtic distribution and $65 \%$ leptokurtic distribution) were obtained. These values of $R_{\mathrm{sk}}>0$ mean deeper-larger amplitude profile valleys in the whole cutting $40 \mathrm{~mm}$ thickness of almost all examined target materials. This reflects the suitable load capacity properties of the machined surface. As the TR increased, the $R_{\mathrm{sk}}$ in the lower half of the cutting thickness (around $\sim 20 \mathrm{~mm}$ ) of almost all studied materials is $R_{\mathrm{sk}}>$ 0 . This shows the amplitude profiles of the machined surface to have more rugged peaks and for these to be more frequent.

Figure 4(c) shows $R_{\mathrm{sk}}$ against $R_{\mathrm{ku}}$ with different AMFR process parameters. It shows that the general trend of positive and negative skewness is almost equally distributed over all variable processes of AMFR and that it is more likely to exhibit leptokurtic distribution and a high degree of peakedness. The trend of $R_{\mathrm{sk}}$ and $R_{\mathrm{ku}}$ is in the region of $-0.6 \leq R_{\mathrm{sk}} \leq 0.7$ (46.2\% negatively skewed distribution and $53.8 \%$ positively skewed distribution) and $2.2 \leq R_{\mathrm{ku}} \leq 4.3$ (33.8\% platykurtic distribution and $66.2 \%$ leptokurtic distribution) with a range of 1.3 and 2.1 for $R_{\mathrm{sk}}$ and $R_{\mathrm{ku}}$, respectively, due to leptokurtic distribution and high degree of peakedness of Carrara marble material generated by high and low AMFR process parameters.

\subsection{Topography Distribution Assessment}

A typical surface cut by AWJ technology can be categorized as having a smooth upper zone and a rough lower zone. Therefore, it is reasonable to investigate both top side and bottom side surface roughness at the same time. In this experiment, the machined surface profile of Carrara marble obtained by Taylor Hobson is shown in Figure 5, and was measured at a distance of $5 \mathrm{~mm}$ from the top (jet entry) and the bottom (jet exit) edge of the machined surface with a constant SOD of $4.0 \mathrm{~mm}$. The cut-off length for the measurement is $0.8 \mathrm{~mm}$. The data generated by the AWJ machine reveal that the cut surface at jet entry region is smooth, $-20 \mu \mathrm{m}<$ surface profile $<+20 \mu \mathrm{m}$ (which is an equally distributed surface profile amplitude with the mean and \pm SD values of $-0.00002 \pm 6.7955$ $\mu \mathrm{m}$ ), and is rough at the jet exit, $-120 \mu \mathrm{m}<$ surface profile $<+120 \mu \mathrm{m}$ (which is an irregularly distributed surface profile amplitude with the mean and \pm SD values of $-0.00006 \pm 48.0723 \mu \mathrm{m}$ ). This is due to the synergetic effect of loss of jet energy and the addition of residual chips combined with abrasive particles when the abrasive waterjet machine is penetrating the Carrara marble sample.

Interestingly, in zone (A) around $5 \mathrm{~mm}$ from the top edge, the data output of the surface topography was found to be dominated $100 \%$ by the kinetic energy generated by the abrasive waterjet machine rather than by any other constant or variable process parameters of the AWJ machine. It revealed that the maximum amplitude approximation of the surface profile in zone (A) for all samples with constant and variable parameters are in the range of $\pm 20 \mu \mathrm{m}$ and exhibit very 

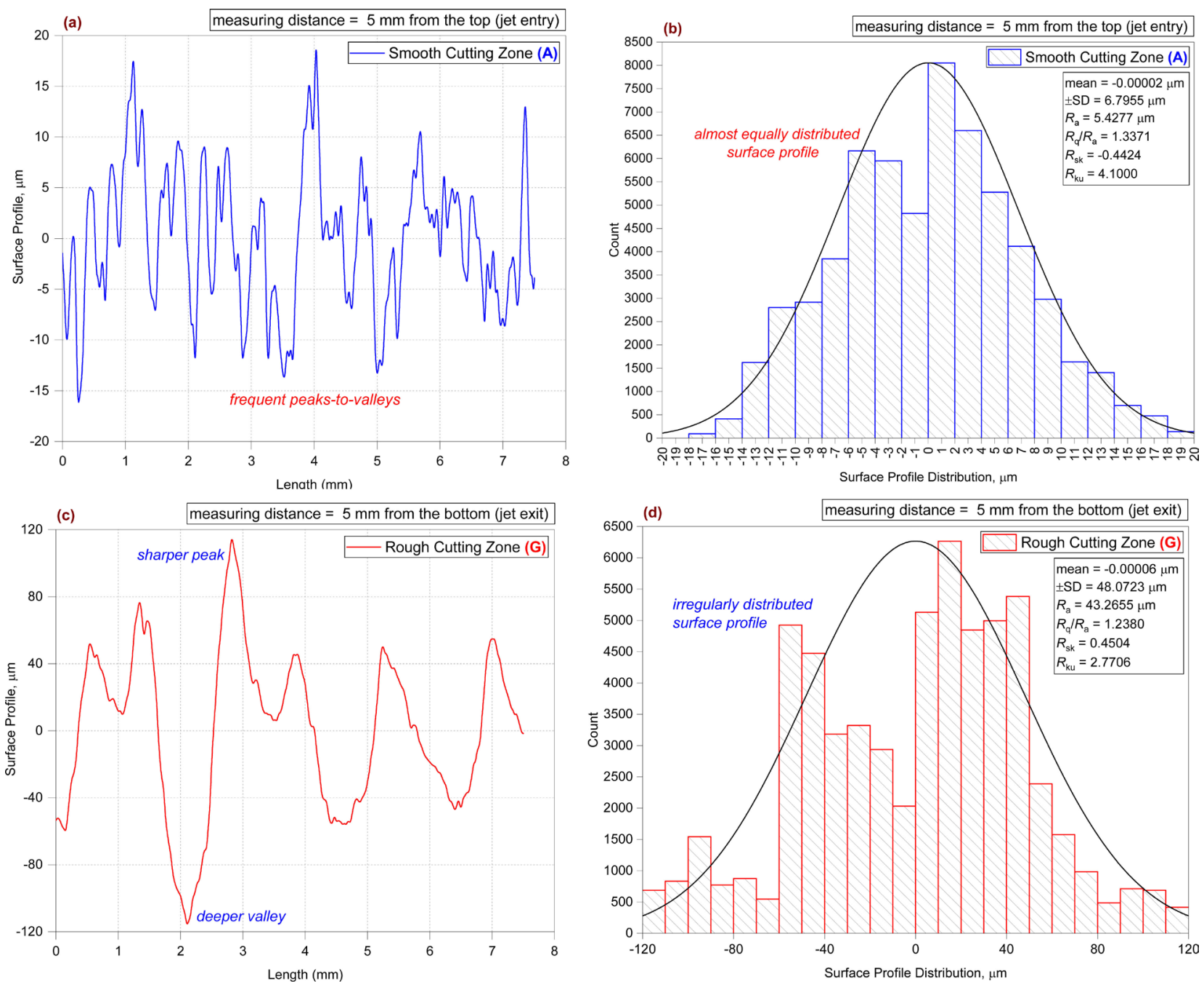

Figure 5. Surface profile of Carrara marble in zone (A) and zone (G).

similar profile height distributions. Whereas, in zone $(\mathrm{G})$ around $5 \mathrm{~mm}$ from the bottom edge, the data output of the surface topography was found to be dominated mostly by constant or variable parameters of the AWJ machine as the kinetic energy no longer exists and the transition zones separate both ranges. Figures 6-8 show the comparative study of surface topography distribution at zone (A) with different SOD, TR, and AMFR. This is clear evidence that even with the various process parameters of the AWJ, yet almost the same amplitude distribution exists in the first zone of the target material.

\subsection{Lagging and WaterJet Angle Assessment}

The lagging (the distance between the jet entry and the jet exit) and waterjet angle of all Carrara marble samples were evaluated and edited by creating a digital photo of the machined surface profile after being cut by the AWJ together with a reference gauge. Then, an image processing technique was used to analyse the data. Also, lines outlining machining marks created by AWJ and showing their estimated lagging and waterjet curve angle were added. Figure 9 shows the 


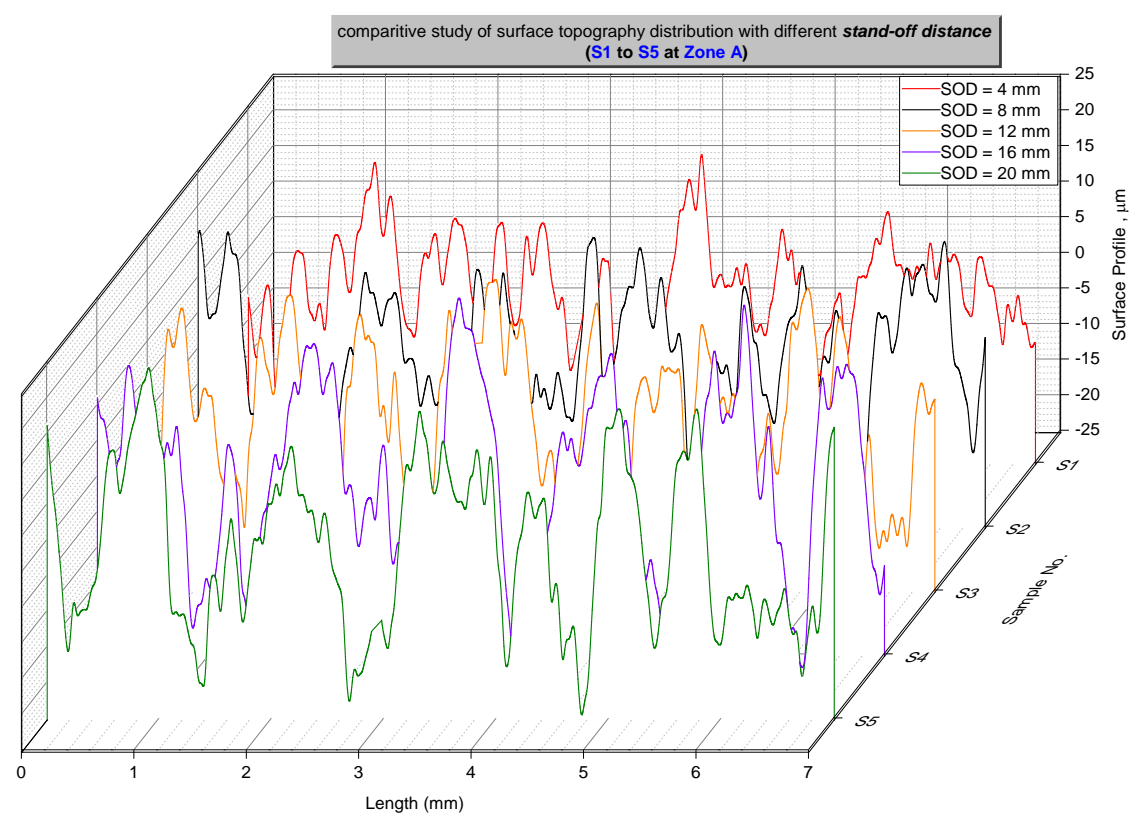

Figure 6. Comparative study of surface topography distribution at zone (A) with different SOD.

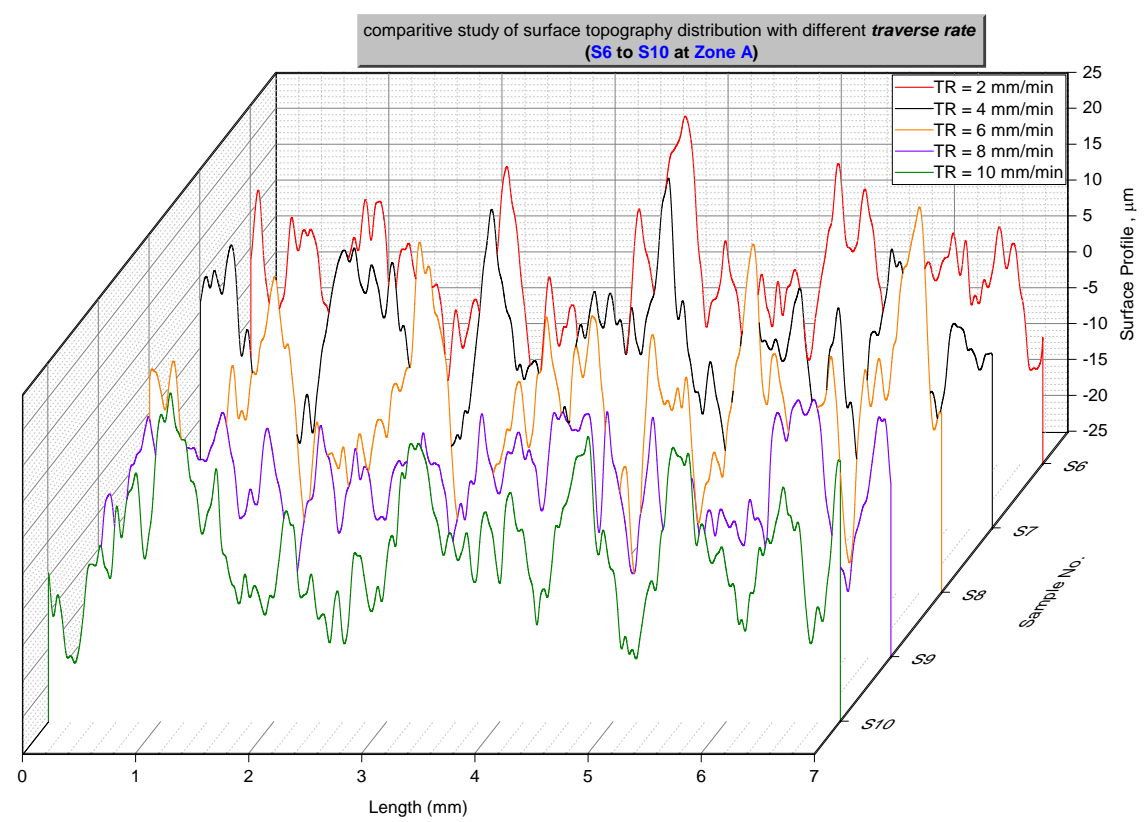

Figure 7. Comparative study of surface topography distribution at zone (A) with different TR.

Carrara marble after being cut and the image processing technique calculating the lagging and the waterjet angle. Besides, Table 1 illustrated the lagging and waterjet angle of all samples after the cutting process. The data output revealed that there was a huge correlation and impact between the variable parameters of AWJ cutting, lagging and waterjet angle. As the SOD increases, the lagging and waterjet angle decreases by $8.85 \%$ and $18.30 \%$, respectively, indicating that the percentage decrease in the waterjet angle is almost double the percentage de- 
crease in the lagging. In contrast, as the TR increases, the lagging and waterjet angle increases by $103.4 \%$ and $49.3 \%$, respectively, indicating that the percentage increase in the waterjet angle is almost half that in the lagging. The results revealed that the SOD affects the waterjet angle more than the lagging with constant TR while the TR affected the lagging more than the waterjet angle at constant SOD. There is almost no difference in lagging for high and low AMFR, but at high AMFR, a low waterjet angle is observed and it appears to be otherwise on the low AMFR with constant SOD and TR.

For the machined surface cut by AWJ with the highest TR, many grooves and elevations in the lower zone are clearly visible. With the decrease in cutting TR, an improvement of machined surface quality in its lower part can be observed.

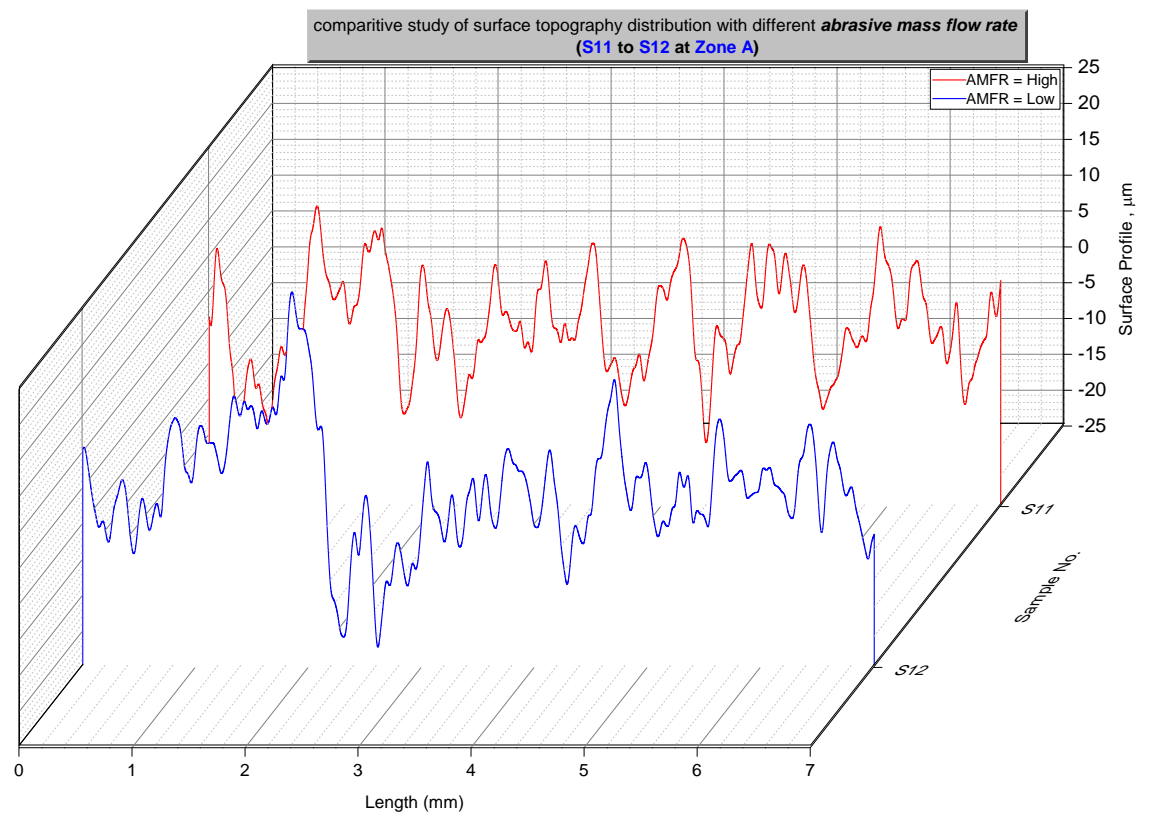

Figure 8. Comparative study of surface topography distribution at zone (A) with different AMFR.

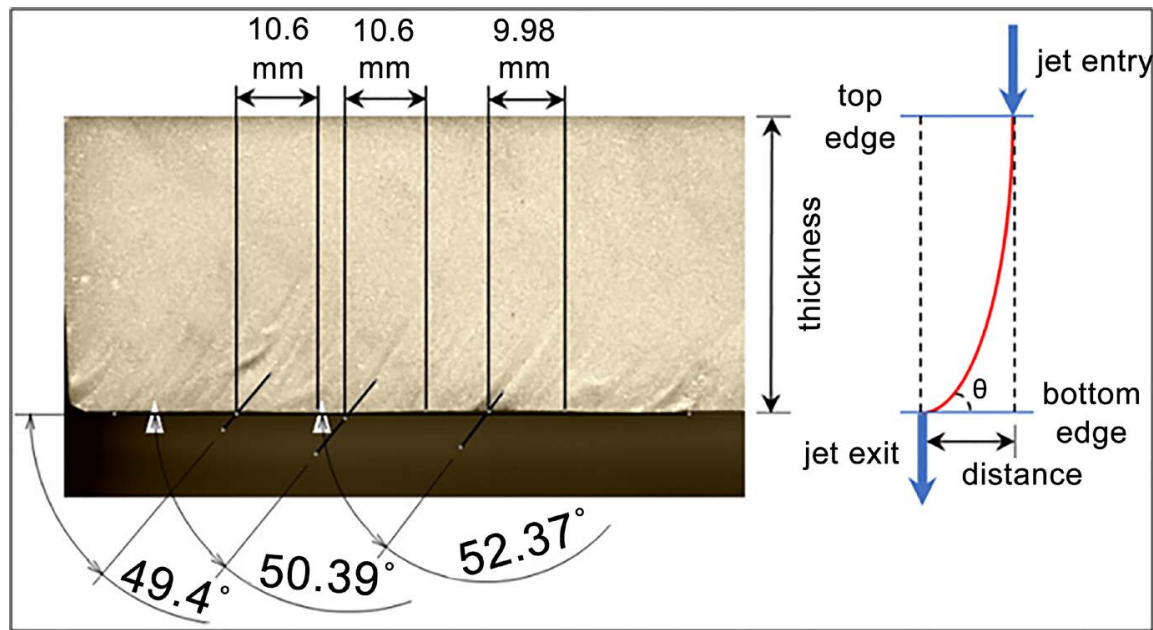

Figure 9. Image processing technique calculating the lagging and the waterjet angle. 
Table 1. Lagging and waterjet curve angle of all Carrara marble samples after AWJ cutting process.

\begin{tabular}{|c|c|c|c|c|c|}
\hline \multirow{2}{*}{$\begin{array}{l}\text { Sample } \\
\text { No. }\end{array}$} & \multicolumn{3}{|c|}{ Process Parameters } & \multirow{2}{*}{$\begin{array}{l}\text { Lagging }(\mathrm{mm}) \\
\text { mean } \pm \mathrm{SD}\end{array}$} & \multirow{2}{*}{$\begin{array}{l}\text { WaterJet Angle }\left({ }^{\circ}\right) \\
\text { mean } \pm \mathrm{SD}\end{array}$} \\
\hline & $\begin{array}{l}\text { SOD } \\
(\mathrm{mm})\end{array}$ & $\begin{array}{c}\text { TR } \\
(\mathrm{mm} / \mathrm{min})\end{array}$ & AMFR & & \\
\hline 1 & 4 & & & $10.39 \pm 0.36$ & $60.09 \pm 0.41$ \\
\hline 2 & 8 & & & $10.23 \pm 0.33$ & $54.44 \pm 5.39$ \\
\hline 3 & 12 & 3 & Medium & $9.93 \pm 0.43$ & $52.55 \pm 6.37$ \\
\hline 4 & 16 & & & $9.89 \pm 0.83$ & $50.72 \pm 1.51$ \\
\hline 5 & 20 & & & $9.47 \pm 0.73$ & $49.04 \pm 1.17$ \\
\hline 6 & & 2 & & $4.66 \pm 0.02$ & $48.71 \pm 0.59$ \\
\hline 7 & & 4 & & $5.13 \pm 0.41$ & $51.05 \pm 1.14$ \\
\hline 8 & 3 & 6 & Medium & $5.36 \pm 0.11$ & $55.13 \pm 0.08$ \\
\hline 9 & & 8 & & $5.59 \pm 0.20$ & $60.38 \pm 0.02$ \\
\hline 10 & & 10 & & $9.48 \pm 0.04$ & $72.71 \pm 0.31$ \\
\hline 11 & 3 & 3 & High & $3.12 \pm 0.04$ & $66.57 \pm 0.31$ \\
\hline 12 & & & Low & $3.52 \pm 0.16$ & $71.48 \pm 0.94$ \\
\hline
\end{tabular}

For the lowest used cutting TR, machining marks are fewer and only faintly visible. It can be noted that the width of the zone with visible machining marks and their curve angle increases with the growth in cutting TR. The presence of machining marks in the lower part of cut surfaces is linked to the decrease in kinetic energy of abrasive particles in the AWJ. Therefore, it can be inferred that with the increase in cutting TR, the kinetic energy of abrasive waterjet drops.

\section{Conclusions}

An investigation of the cutting performance and erosive process in abrasive waterjet (AWJ) machining of Carrara marble material is presented. It demonstrates that AWJ cutting can generate a good machined surface quality at high production rates if the cutting parameters are suitably selected. The abrasive waterjet process parameters were successfully employed in contour machining of up to $40 \mathrm{~mm}$ thick Carrara marble that is being used in Al-Masjid Al-Haram, The Holy Mosque, in Makkah, KSA, for internal flooring and internal/external cladding application. The conclusions were as follows:

The abrasive waterjet process was characterized in terms of an $R_{\mathrm{q}} / R_{\mathrm{a}}$ ratio and was determined to be $R_{\mathrm{q}} / R_{\mathrm{a}}=1.23 \pm 0.04$ for SOD, $R_{\mathrm{q}} / R_{\mathrm{a}}=1.25 \pm 0.03$ for TR and $R_{\mathrm{q}} / R_{\mathrm{a}}=1.26 \pm 0.04$ for AMFR.

The values of $R_{\mathrm{sk}}$ and $R_{\mathrm{ku}}$ are strongly influenced by AWJ on the machined surface. Oppositely signed values for $R_{\mathrm{sk}}$ correspond to different machined surfaces, while different values of $R_{\mathrm{ku}}$ reveal different machined surface features.

The data generated by AWJ machining showed that there was a huge correlation between the SOD, TR and AMFR process parameters and the lagging and waterjet angle. 
To sum up, SOD and TR are the key process parameters that affect the machined surface quality of Carrara marble in contrast to AMFR.

\section{Acknowledgements}

The authors would like to express their sincere thanks and appreciation for the project of the Custodian of the Two Holy Mosques King Abdullah Bin Abd Al-Aziz for Mataf Extension providing Carrara marble as workpiece materials for this investigation.

\section{Conflicts of Interest}

The authors have no conflicts of interest.

\section{Funding}

The authors received no financial support for the research and/or for the publication of this article.

\section{References}

[1] Rusnák, J., et al. (2011) Measurement of Titanium Surface Roughness Created by Non-Conventional Cutting Technology. Research in Agricultural Engineering, 57, S57-S60.

[2] Jiang, X., et al. (2007) Paradigm Shifts in Surface Metrology. Part I. Historical Philosophy. Proceedings of the Royal Society A: Mathematical, Physical and Engineering Science, 463, 2049-2070. https://doi.org/10.1098/rspa.2007.1874

[3] Momber, A.W. and Kovacevic, R. (1998) Principles of Abrasive Water Jet Machining. Springer-Verlag, London. https://doi.org/10.1007/978-1-4471-1572-4

[4] Momber, A.W. and Kovacevic, R. (1995) Quantification of Energy Absorption Capability in Abrasive Water Jet Machining. Proceedings of the Institution of Mechanical Engineers, Part B: Journal of Engineering Manufacture, 209, 491-498. https://doi.org/10.1243/PIME_PROC_1995_209_113_02

[5] Klichova, D. and Klich, J. (2016) Study of the Effect of Material Machinability on Quality of Surface Created by Abrasive Water Jet. Procedia Engineering, 149, 177 182.

[6] Alsoufi, M.S. (2017) State-of-the-Art in Abrasive Water Jet Cutting Technology and the Promise for Micro- and Nano-Machining. International Journal of Mechanical Engineering and Applications, 5, 1-14. https://doi.org/10.11648/j.ijmea.20170501.11

[7] Pal, V.K. and Choudhury, S.K. (2016) Fabrication of Texturing Tool to Produce Array of Square Holes for EDM by Abrasive Water Jet Machining. The International Journal of Advanced Manufacturing Technology, 85, 2061-2071. https://doi.org/10.1007/s00170-015-7875-7

[8] Syazwani, H., Mebrahitom, G. and Azmir, A. (2016) A Review on Nozzle Wear in Abrasive Water Jet Machining Application. IOP Conference Series: Materials Science and Engineering, 114, Article ID: 012020. https://doi.org/10.1088/1757-899X/114/1/012020

[9] Dong, Y., et al. (2014) On-Line Recycling of Abrasives in Abrasive Water Jet Cleaning. Procedia CIRP, 15, 278-282.

[10] Kartal, F. (2017) A Review of the Current State of Abrasive Water-Jet Turning Ma- 
chining Method. The International Journal of Advanced Manufacturing Technology, 88, 495-505. https://doi.org/10.1007/s00170-016-8777-z

[11] Zeleňák, M., et al. (2012) Comparison of Surface Roughness Quality Created by Abrasive Water Jet and CO2 Laser Beam Cutting. Tehnickivjesnik, 19, 481-485.

[12] Begic-Hajdarevic, D., et al. (2015) Experimental Study on Surface Roughness in Abrasive Water Jet Cutting. Procedia Engineering, 100, 394-399.

[13] Löschner, P., Jarosz, K. and Niesłony, P. (2016) Investigation of the Effect of Cutting Speed on Surface Quality in Abrasive Water Jet Cutting of 316L Stainless Steel. Procedia Engineering, 149, 276-282.

[14] ISO4287 (1997) Geometrical Product Specifications (GPS)—Surface Texture: Profile Method-Terms, Definitions and Surface Texture Parameters. ISO.

[15] Alsoufi, M.S. and Bawazeer, T.M. (2015) Quantifying Assessment of Touch-Feel Perception: An Investigation Using Stylus Base Equipment and Self-Touch (Human Fingertip). Journal of Engineering and Architecture, 1, 1-16.

[16] Alsoufi, M.S. and Bawazeer, T.M. (2015) The Effect of Aggressive Biological Materials on a Painted Automotive Body Surface Roughness. American Journal of Nano Research and Applications, 3, 17-26.

[17] Suker, D.K., et al. (2016) Studying the Effect of Cutting Conditions in Turning Process on Surface Roughness for Different Materials. World Journal of Research and Review, 2, 16-21.

[18] Alsoufi, M.S., et al. (2016) Experimental Study of Surface Roughness and Micro-Hardness Obtained by Cutting Carbon Steel with Abrasive Water Jet and Laser Beam Technologies. American Journal of Mechanical Engineering, 4, 173-181.

[19] Bawazeer, T.M., et al. (2016) Effect of Aqueous Extracts of Salvadora Persica "Miswak" on the Acid Eroded Enamel Surface at Nano-Mechanical Scale. Materials Sciences and Applications, 7, 754-771. https://doi.org/10.4236/msa.2016.711059

[20] Alsoufi, M.S., et al. (2016) Surface Roughness and Knoop Indentation Micro-Hardness Behavior of Aluminium Oxide $\left(\mathrm{Al}_{2} \mathrm{O}_{3}\right)$ and Polystyrene $\left(\mathrm{C}_{8} \mathrm{H}_{8}\right)_{\mathrm{n}} \mathrm{Ma}$ terials. International Journal of Mechanical \& Mechatronics Engineering, 16, 43-49.

[21] Dong, W.P., Sullivan, P.J. and Stout, K.J. (1994) Comprehensive Study of Parameters for Characterising Three-Dimensional Surface Topography: III: Parameters for Characterising Amplitude and Some Functional Properties. Wear, 178, 29-43.

[22] Dong, W.P., Sullivan, P.J. and Stout, K.J. (1994) Comprehensive Study of Parameters for Characterising Three-Dimensional Surface Topography: IV: Parameters for Characterising Spatial and Hybrid Properties. Wear, 178, 45-60.

[23] Dong, W.P., Sullivan, P.J. and Stout, K.J. (1992) Comprehensive Study of Parameters for Characterizing Three-Dimensional Surface Topography I: Some Inherent Properties of Parameter Variation. Wear, 159, 161-171.

[24] Dong, W.P., Sullivan, P.J. and Stout, K.J. (1993) Comprehensive Study of Parameters for Characterizing Three-Dimensional Surface Topography II: Statistical Properties of Parameter Variation. Wear, 167, 9-21.

[25] Alsoufi, M.S., et al. (2017) Influence of Abrasive Water Jet Machining Parameters on the Surface Texture Quality of Carrara Marble. Journal of Surface Engineered Materials and Advanced Technology, 7, 25-37. https://doi.org/10.4236/jsemat.2017.72003

[26] Klichova, D., Klich, J. and Gurkova, L. (2016) Study of Quality of Nine Aluminium Alloys Surfaces Created Using Abrasiv Water Jet. Modern Machinery Science Journal, 892-895. 
Submit or recommend next manuscript to SCIRP and we will provide best service for you:

Accepting pre-submission inquiries through Email, Facebook, LinkedIn, Twitter, etc. A wide selection of journals (inclusive of 9 subjects, more than 200 journals)

Providing 24-hour high-quality service

User-friendly online submission system

Fair and swift peer-review system

Efficient typesetting and proofreading procedure

Display of the result of downloads and visits, as well as the number of cited articles Maximum dissemination of your research work

Submit your manuscript at: http://papersubmission.scirp.org/

Or contact msa@scirp.org 